\title{
State of the art in magnetic resonance imaging of hepatocellular carcinoma
}

\author{
Natally Horvat ${ }^{1,2,3}$, Serena Monti ${ }^{4}$, Brunna Clemente Oliveira2,3, \\ Camila Carlos Tavares Rocha ${ }^{3}$, Romina Grazia Giancipoli ${ }^{5}$, Lorenzo Mannelli ${ }^{1}$ \\ 1 Department of Radiology, Memorial Sloan Kettering Cancer Center, New York, USA \\ ${ }^{2}$ Department of Radiology, Hospital Sírio-Libanês, São Paulo, Brazil \\ ${ }^{3}$ Department of Radiology, Hospital das Clínicas da Faculdade de Medicina da Universidade de São Paulo, \\ Dr. Enéas de Carvalho Aguiar, São Paulo, Brazil \\ ${ }^{4}$ IRCCS SDN, Naples, Italy \\ ${ }^{5}$ Department of Nuclear Medicine, Sapienza University of Rome, Roma, Itália
}

Radiol Oncol 2018; 52(4): 353-364.

Received 2 October 2018

Accepted 19 October 2018

Correspondence to: Lorenzo Mannelli, M.D., Ph.D., Department of Radiology, Memorial Sloan Kettering Cancer Center. 300 East 66 th Street, New York, NY, 10021, USA. Phone: +1 646-888-541; Fax: +1 929-321-5013; E-mail: mannellilorenzo@yahoo.it

Disclosure: No potential conflicts of interest were disclosed.

Background. Liver cancer is the sixth most common cancer worldwide and the second leading cause of cancer mortality. Chronic liver disease caused by viral infection, alcohol abuse, or other factors can lead to cirrhosis. Cirrhosis is the most important clinical risk factor for hepatocellular carcinoma $(\mathrm{HCC})$ whereby the normal hepatic architecture is replaced by fibrous septa and a spectrum of nodules ranging from benign regenerative nodules to HCC, each one of them with different imaging features.

Multiple studies have demonstrated that magnetic resonance imaging (MRI) has excellent sensitivity and specificity for the detection and characterization of HCC in comparison with computed tomography (CT) and ultrasound. Beyond the standard protocol, the use of hepatobiliary contrast agents and the acquisition of additional sequences such as diffusion weighted imaging (DWI) with apparent diffusion coefficient mapping, subtraction imaging, multiplanar acquisition, and hepatobiliary phase, have been proposed to improve the detection of HCC, especially in the case of small, well-differentiated, and post-treatment HCC.

Conclusions. Furthermore, advanced techniques including the quantification of hepatic and intralesional fat and iron, magnetic resonance elastography, radiomics, radiogenomics, and positron emission tomography (PET)-MRI are highly promising for the extraction of new imaging biomarkers that reflect the tumor microenvironment and, in the future, may add decision-making value in the management of patients with HCC.

Key words: hepatocellular carcinoma; hepatic nodule; liver; cirrhosis; magnetic resonance imaging.

\section{Introduction}

Liver cancer is the sixth most common cancer worldwide and the second leading cause of cancer mortality. ${ }^{1,2}$ In the United States, approximately 42220 new cases of liver cancer will be diagnosed and 30200 deaths will occur in $2018 .^{3}$ The incidence in men is three times higher than in women. Over a third of liver cancer consists of hepatocellular car- cinoma (HCC). In recent years, five-year survival rates of HCC have considerably improved due to earlier detection and curative therapies. ${ }^{4}$ However, the incidence is still rising in women while it has reached a plateau in men since $2010 .^{3}$

The two most common risk factors of HCC are chronic infection from hepatitis B and/or hepatitis $\mathrm{C}$ virus and alcohol abuse. ${ }^{3}$ Other important risk factors include consumption of aflatoxin (toxin 


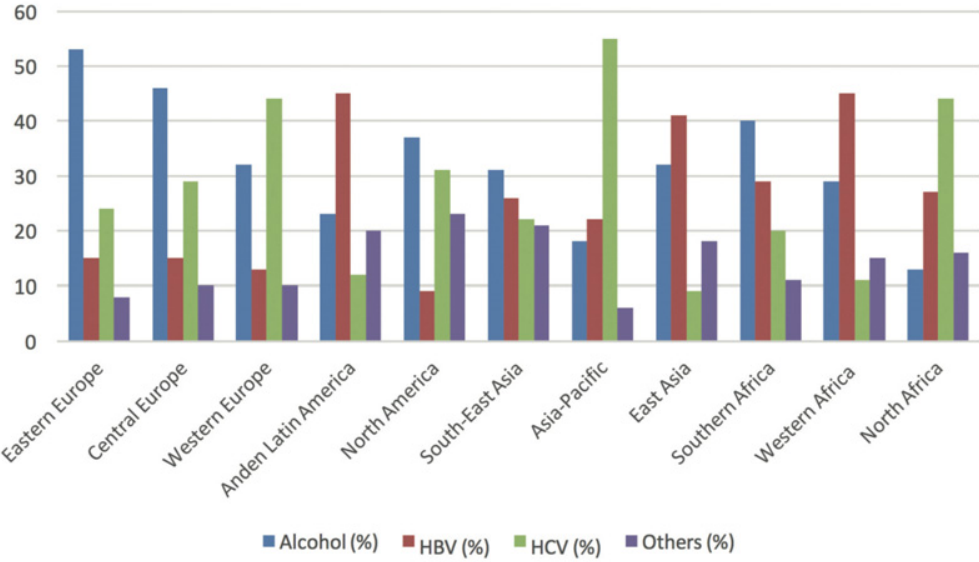

FIGURE 1. Geographical distribution of main risk factors for hepatocellular carcinoma (HCC) worldwide.

produced by a fungus that can infects grains, soybeans and peanuts) which occurs mainly in less developed countries and nonalcoholic fatty liver disease which occurs mainly in Western countries. ${ }^{2}$ Figure 1 demonstrates the geographical distribution of the main risk factors for HCC worldwide.

Chronic liver disease caused by viral infection, alcohol abuse, or other factors can lead to cirrhosis. Cirrhosis is the most important clinical risk factor for HCC whereby the normal hepatic architecture is replaced by fibrous septa and a spectrum of nodules ranging from benign regenerative nodules to HCC. ${ }^{5}$ The cirrhotic liver gives way to HCC via hepatocarcinogenesis, an anaplastic complex process characterized by stepwise accumulation of epigenetic and genetic alterations at the molecular and cellular level ${ }^{6,7}$ and changes in the hepatic architecture seen at the histologic level. While initial-

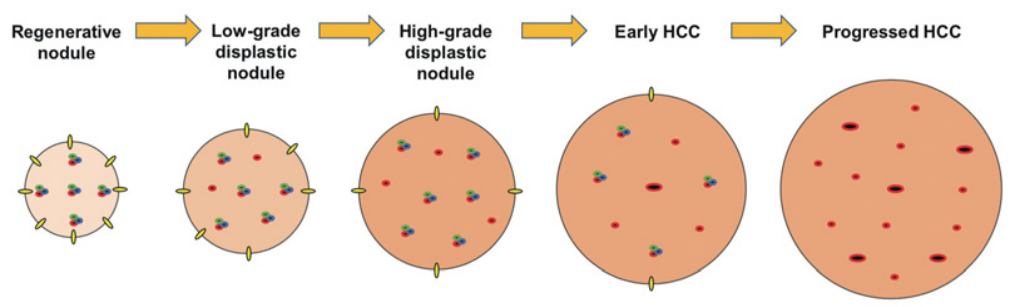

Late Arterial hyperenhancement

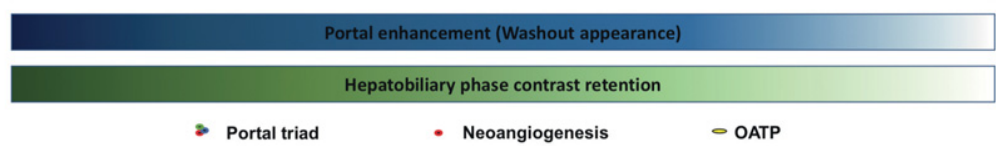

FIGURE 2. Graphic demonstrating hepatocarcinogenesis from regenerative nodules to progressed hepatocellular carcinoma ( $\mathrm{HCC})$, emphasizing the proportion of portal triad, neoangiogenesis, and organic anionic transporting polypeptides, and, consequently, the pattern of enhancement on late arterial, portal, and hepatobiliary phases. ly hepatocarcinogenesis involves the replacement of normal hepatic architecture with regenerative nodules, this subsequently progresses to replacement with dysplastic nodules and then HCC. HCC itself progresses from well-differentiated to poorly differentiated HCC. ${ }^{6}$

The liver has a dual blood supply, i.e., the hepatic portal vein and the hepatic artery. In the normal liver, approximately $75 \%$ of the liver is supplied by the hepatic portal vein. However, during hepatocarcinogenesis, neoangiogenesis decreases the portal blood supply and increases the arterial supply relative to the degree of malignancy within the nodules. This allows lesions to be detected on imaging. Emerging evidence also suggests that in hepatocarcinogenesis, the expression levels of organic anionic transporting polypeptides (OATP), a bile salt transporter protein on hepatocytes membranes, diminishes even before neoangiogenesis, which may have implications for earlier radiological detection of lesions using hepatobiliary agents (Figure 2). ${ }^{7}$

\section{Magnetic resonance imaging}

Magnetic resonance imaging (MRI) has rapidly evolved as a superior imaging technique in the oncologic field in the past few decades, having undergone improvements in its acquisition time and imaging quality. Multiple studies have demonstrated that MRI has excellent sensitivity and specificity for the detection and characterization of HCC compared with computed tomography (CT) and ultrasound.-11 However, mainly because of its high cost and limited availability especially in underdeveloped countries which bear a disproportionately high risk of HCC, its large-scale use for HCC screening is still restricted. Table 1 summarizes the main indications of MRI for HCC as recommended by the European Association (EASL) and American Association for the Study od Liver Diseases (AASLD) guidelines. 9,12

\section{MRI protocol}

Standardized MRI protocols for HCC surveillance must be developed to allow clinicians and technologists to perform repeatable and reproducible high-quality examinations. ${ }^{11}$ The minimum magnetic field strength of 1.5 Tesla (T) provides acceptable temporal, spatial, and contrast resolution that enable adequate assessment of hepatic lesions. ${ }^{12}$ 
The following sequences are essential for the diagnosis of HCC: T2-weighted imaging (WI); unenhanced T1WI opposed and in-phase; and multiphase T1WI (pre-contrast, late arterial, portal venous, and delayed or transitional phases). Slice thickness should be $5 \mathrm{~mm}$ or less for dynamic series and $8 \mathrm{~mm}$ or less for other imaging. ${ }^{13,14}$

Other sequences have been proposed to improve the detection of HCC, especially in cases of well-differentiated HCC or HCC post-treatment. These include diffusion weighted imaging (DWI) with apparent diffusion coefficient mapping, subtraction imaging, multi-planar acquisition, and hepatobiliary phase. ${ }^{14,15}$ Table 2 summarizes these MRI sequences.

DWI improves the characterization of liver nodules without requiring contrast media injection. ${ }^{15,17}$ Currently, DWI is used to increase the sensitivity of other sequences for the detection and characterization of HCC. Studies investigating the utility of DWI have demonstrated promising results for assessing prognosis, predicting response, distinguishing tumor from treatment effect, and monitoring response to therapy in patients with HCC. ${ }^{18-}$ 22

\section{Contrast media agents}

Dynamic contrast-enhanced sequences are routinely performed with gadolinium-based extracellular contrast agents (ECA) or hepatobiliary contrast agents (HBA). They allow the diagnosis of HCC by exploiting the physiologic changes in blood flow that accompany hepatocarcinogenesis. Following
TABLE 1. Main indications of MRI for hepatocellular carcinoma (HCC)

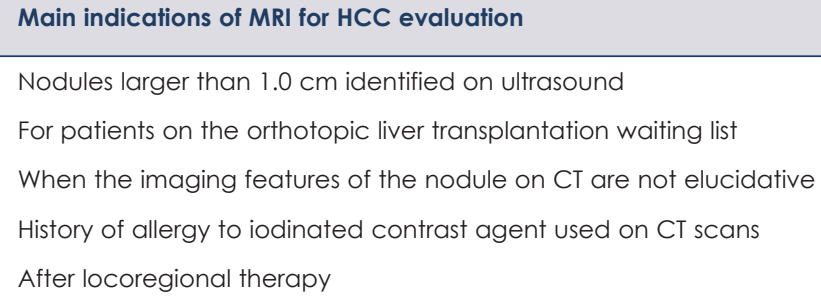

administration of the contrast agent, the dual vascular supply of the liver is opacified in the following sequential order: the hepatic arteries, the portal veins, and finally the hepatic veins. ${ }^{7,14}$

Typically, contrast agents are administered at rates of $2 \mathrm{~mL} / \mathrm{sec}$ followed by saline infusion. The dose is usually based on body weight (ranging from 0.025 to $0.1 \mathrm{mmol}$ gadolinium per $\mathrm{kg}$ ) as well as on the agent and other factors. ${ }^{7}$

ECAs include gadoterate meglumine (GdDOTA) and gadopentate dimeglumine (Gd-DTPA) which are excreted primarily through glomerular filtration. The pattern of contrast enhancement can be studied in dynamic T1WI in the following phases: late arterial, portal venous, and delayed phases. HBAs include gadoxetate disodium (GdEOB-DTPA) and gadobenate dimeglumine (GdBOPTA) which are excreted through glomerular filtration but are also taken up by hepatocytes and excreted into the biliary system. As such, HBAs provide additional information regarding the presence of hepatocytes with OATP, which decrease in hepatocarcinogenesis. ${ }^{23}$ With HBA administration,

TABLE 2. MRI sequences and hepatocellular carcinoma (HCC) features that can be assessed in each sequence

\begin{tabular}{|c|c|}
\hline MRI sequences & HCC imaging features \\
\hline T2WI & Usually hyperintense \\
\hline TIWI opposed and in-phase & Intralesional microscopic fat (lower signal on opposed-phase) or iron (lower signal on in-phase) \\
\hline T1WI with fat saturation pre-contrast & $\begin{array}{l}\text { Demonstrates the presence of macroscopic fat and blood products } \\
\text { After locoregional therapies, hyperintensity indicates coagulative necrosis }\end{array}$ \\
\hline Dynamic late arterial phase & Hyperenhancement \\
\hline Dynamic portal venous phase & Washout and capsule appearance \\
\hline Dynamic delayed phase & Washout and capsule appearance \\
\hline Diffusion weighted imaging & Restricted diffusion (helps to identify small lesions) \\
\hline Subtraction imaging & $\begin{array}{l}\text { Characterizes contrast enhancement in spontaneously hyperintense nodules on TIWI pre-contrast } \\
\text { (especially important for lesions with blood products and after locoregional treatment) }\end{array}$ \\
\hline Multi-planar acquisition & Helps to differentiate HCC from mass-like lesions or extra-hepatic lesions \\
\hline Hepatobiliary phase & Generally hypointense \\
\hline
\end{tabular}

$\mathrm{T} 1 \mathrm{~W} 1=\mathrm{T} 1$ weighted image; $\mathrm{T} 2 \mathrm{~W} 1=\mathrm{T} 2$ weighted image 
the pattern of contrast enhancement can be studied in the late arterial, portal venous, transitional, and hepatobiliary phases.

Recently, in Europe, the use of intravenous linear agents such as gadodiamide and intravenous gadopentate dimeglumine have been suspended or restricted in response to the retention of gadolinium in the brain and in other tissues as reported in a scientific review. ${ }^{24}$ However, there is still no evidence that this deposition causes any harm to patients. Hepatobiliary linear contrast agents continue to be available as their properties allow the recognition of poorly vascularized hepatic lesions which cannot be studied with other agents. The macrocyclic agents (gadobutrol, gadoteric acid, and gadoteridol) have a lower propensity to release gadolinium than linear agents and can continue to be used in their current indications. ${ }^{24}$

\section{Advanced techniques}

\section{Quantification of fat and iron}

Liver biopsy is the gold standard for quantifying iron and fat in the liver. However, this method is invasive and susceptible to sampling variability. MRI is a non-invasive, alternative method to quantify iron and fat within the liver. There are several sequences that can be used to quantify iron and fat within the liver. Regarding iron, the following techniques can be employed: signal intensity ratio techniques based on T2WI or T2 ${ }^{*} \mathrm{WI}$, quantitative relaxometry (based mainly on T2WI but also on T1WI), and MR susceptometry. ${ }^{25-26}$ In regards to fat, postprocessing of T1WI in- and opposed-phase provides quantification from a scale of $0-50 \%$ while the proton density fat fraction allows quantification of a full fat fraction from $0-100 \% .{ }^{27}$ Iron and fat content may contribute to differential diagnosis and may be a potential prognostic biomarker of HCC. ${ }^{28}$ However, the evidence is still limited and this method is not used in the daily practice.

\section{Magnetic resonance elastography}

Magnetic Resonance Elastography (MRE) is an imaging modality used to stage liver fibrosis..$^{29,30}$ Recent studies have demonstrated the use of MRE for the evaluation of HCC with promising results for the prediction of tumor grade ${ }^{31}$ and assessment of treatment response. ${ }^{32}$ Well/moderately differentiated tumors demonstrated increased stiffness compared to poorly differentiated ones and there was a correlation between the percentage of tumor necrosis and tumor stiffness, particularly in HCCs treated with radioembolization. ${ }^{31}$ However, the evidence is still limited and MRE is not yet routinely implemented.

\section{Imaging features on MRI}

\section{Regenerative nodules}

Regenerative nodules correspond to an area of parenchyma surrounded by fibrosis. These nodules are usually similar to background liver parenchyma but some may exhibit a fine area of peripheral late-phase enhancement corresponding to fibrosis. Regenerative nodules may present accumulation of iron which results in low signal intensity on T1and T2-weighted imaging. .,32 $^{6}$

\section{Dysplastic nodules}

Dysplastic nodules contain atypical cells but without malignancy on histological analysis. The radiological pattern of dysplastic nodules is variable and can be similar to regenerative nodules (in those with low-grade dysplasia) or well-differentiated HCC (in those with high-grade dysplasia). ${ }^{33-35}$ These lesions often present as iso- or hypointense on T2-weighted imaging and are frequently hypovascular. Occasionally, a mildly elevated signal intensity may occur within a low signal-intensity nodule on T2-weighted imaging. This represents foci of HCC (the foci of high signal intensity) within a dysplastic nodule (the area with low signal intensity). The foci of HCC may also enhance in the arterial phase. ${ }^{35}$

\section{HCC}

HCC has a wide spectrum of radiological characteristics depending on its size and degree of histological differentiation. HCC can be classified as early or progressed HCC.

Early HCC often measures less than $2.0 \mathrm{~cm}$ and sometimes appears similar to high-grade dysplastic nodules on imaging. Histologically, it differs from dysplastic nodules because of stromal invasion. Radiologically, it has higher T2WI signal intensity, hypo- or iso- vascularization in the arterial phase, and washout appearance in the delayed phase. ${ }^{7}$ Mild restricted diffusion has improved the sensitivity for HCC detection, mostly for small HCC, especially well-differentiated HCC with atypical postcontrast imaging patterns. ${ }^{36}$ 
Progressed HCCs are malignant lesions with the ability to invade vascular planes and metastasize. The radiological pattern is variable, but frequently a mosaic pattern is exhibited due to nodular areas' being interspersed by areas of hemorrhage, arteriovenous shunting, fibrosis, and necrosis. The main findings are: high signal intensity on T2-weighted imaging (Figures 3-4), hyperenhancement on arterial phase (Figures 3-7), washout appearance on delayed phase (Figures 3-5), and nodules that are surrounded by a capsule / pseudocapsule (more evident in the delayed phase) (Figure 3).

HCC may also be classified according to its growth patterns / macroscopic appearance into: single nodular type, well-defined, encapsulated with better prognosis; or multifocal type (multiple nodules in several hepatic segments), with a diffuse pattern, usually extensive, heterogeneous, with variable enhancement, usually better detected in the delayed phase (hypoenhancement), and often associated with vascular invasion (Figure 4). ${ }^{35-37}$ Table 3 summarizes the main imaging features of HCC.

\section{Atypical HCC}

A minority of HCC presents atypical imaging features and awareness of these is important for early
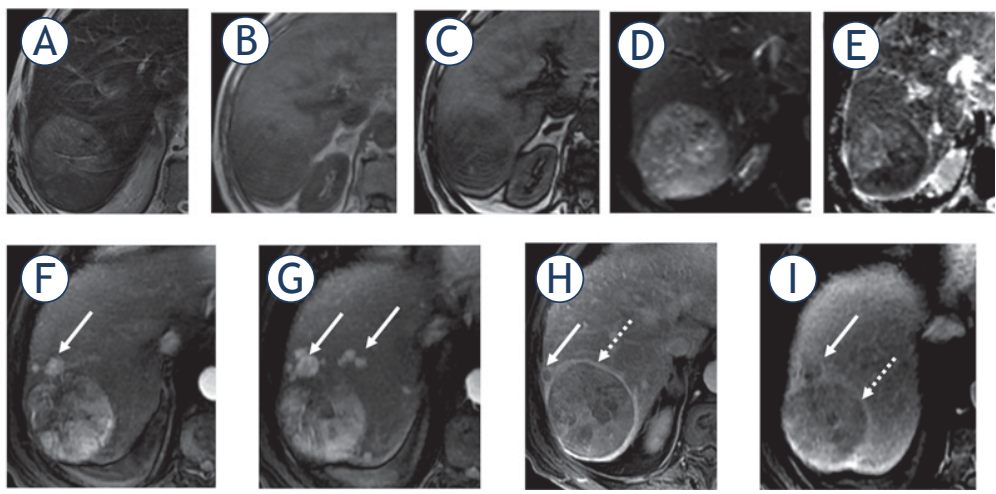

FIGURE 3. 53-year-old man with cirrhosis due to hepatitis $B$ and hepatocellular carcinoma. Hepatic nodule in segment VII with high signal intensity on T2 weighted image (T2WI) (A); with fat content (B, C) demonstrated by reduction of signal intensity on $\mathrm{Tl}$ weighted image (TIWI) opposite phase $(C)$ when compared with TIWI in-phase (B); restriction on diffusion (D, E) characterized by high signal on diffusion weighted imaging (DWI) (D) and low signal on the apparent diffusion coefficients (ADC) map (E). On dynamic phases ( $F-I)$, the nodule showed arterial hypervascular enhancement in the late arterial phase (F); mosaic architecture (F-I); washout appearance in the portal venous (H) and delayed (I) phases; and capsule (dashed arrows) (H-I) and satellites nodules (arrows) with the same pattern of enhancement (F-I).

TABLE 3. Main imaging features of hepatocellular carcinoma (HCC)

\begin{tabular}{|c|c|}
\hline Imaging features & Description \\
\hline Arterial hyperenhancement & Increased enhancement in the arterial phase. Reflects tumor neoangiogenesis. \\
\hline Washout appearance & $\begin{array}{l}\text { Hypoenhancement of the lesion compared with background liver tissue. Secondary to HCC extracellular } \\
\text { reduced volume, rapid venous drainage and reduced intranodular portal venous supply. }\end{array}$ \\
\hline Capsule appearance & $\begin{array}{l}\text { Observed in approximately } 80 \% \text { of } \mathrm{HCCS} \text {, detected on delayed phase, secondary to the lack of portal } \\
\text { supply to malignant nodules. Corresponds to a pseudocapsule consisting of compressed adjacent liver } \\
\text { parenchyma with occasional nonspecific inflammatory cells on histology. }\end{array}$ \\
\hline Portal vein tumoral thrombosis & $\begin{array}{l}\text { HCC invades and grows within the lumen. The vein appears dilated and with the same pattern of } \\
\text { enhancement observed in the nodule. }\end{array}$ \\
\hline T2 hyperintensity & The elevated signal intensity on T2WI can be useful to differentiate HCC from dysplastic nodules. \\
\hline Restricted diffusion & $\begin{array}{l}\text { Mildly elevated signal relative to the surrounding liver parenchyma on diffusion weighted imaging (DWI) } \\
\text { and low signal intensity on apparent diffusion coefficients (ADC) map. }\end{array}$ \\
\hline Corona enhancement & $\begin{array}{l}\text { Enhancement of the peritumoral parenchyma after enhancement of the tumor itself, because of the } \\
\text { passage of contrast through the draining sinusoids and portal venules into the surrounding sinusoids. }\end{array}$ \\
\hline Intralesional fat & Loss of signal on the opposed-phase TIWI compared with the in-phase images. \\
\hline Lesion iron sparing & $\begin{array}{l}\text { Siderotic nodule is likely to be a dysplastic nodule. Development of an iron-free around the nodule } \\
\text { suggests HCC foci. }\end{array}$ \\
\hline Mosaic architecture & $\begin{array}{l}\text { Nodular areas interspersed by areas of fibrosis, hemorrhage, arteriovenous shunting and necrosis. } \\
\text { Characteristic of progressed HCCs. }\end{array}$ \\
\hline Nodule-in-nodule architecture & $\begin{array}{l}\text { Mildly elevated signal intensity on T2WI within nodule with low signal intensity, representing the focus of } \\
\text { HCC within the low density dysplastic nodule, that may also enhance in the arterial phase. }\end{array}$ \\
\hline Transitional phase hypointensity & $\begin{array}{l}\text { Hypointensity compared with background liver following administration of a hepatobiliary contrast agent } \\
\text { (2-5 minutes after contrast media administration). }\end{array}$ \\
\hline Hepatobiliary phase hypointensity & $\begin{array}{l}\text { Hypointensity compared with background liver following administration of a hepatobiliary contrast agent } \\
\text { (20 minutes after). }\end{array}$ \\
\hline
\end{tabular}

$\mathrm{T} 1 \mathrm{~W} 1=\mathrm{T} 1$ weighted image; $\mathrm{T} 2 \mathrm{Wl}=\mathrm{T} 2$ weighted image 

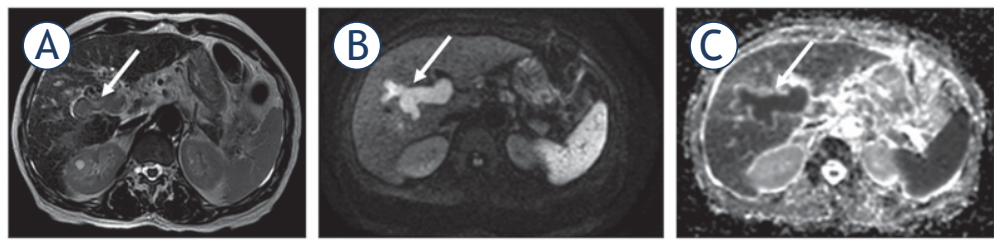

FIGURE 4. 63-year-old man with chronic hepatitis C and HCC with tumor invasion within the portal vein. Main and right portal veins demonstrate dilation (arrows), high signal intensity on $\mathrm{T} 2$ weighted image (T2WI) (A), restriction on diffusion weighted imaging (DWI) $(B, C)$.
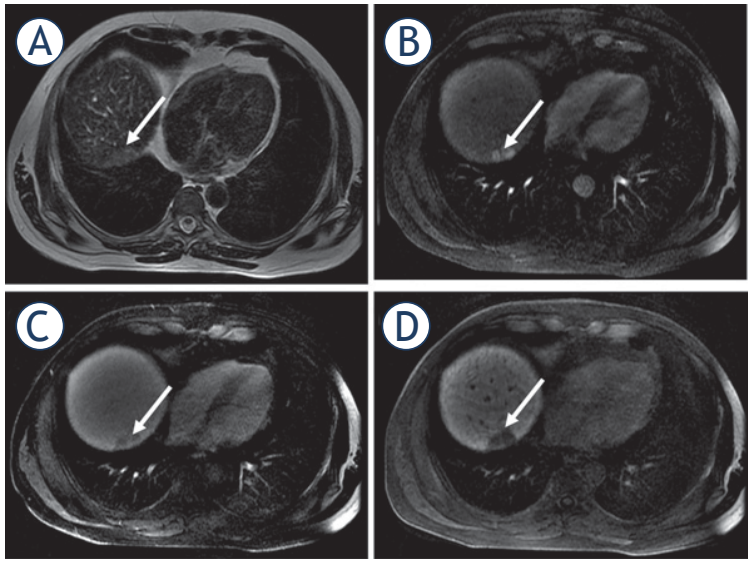

FIGURE 5. 49-year-old woman with cirrhosis due to chronic hepatitis $C$ had a new hepatic nodule detected on screening ultrasound which was confirmed as $\mathrm{HCC}$ on MRI with hepatobiliary contrast agent. Hepatic nodule in segment VII (arrows) with high signal intensity on T2 weighted image (T2WI) (A), hyperenhancement in the late arterial phase (B), washout appearance $(C)$, and hypointense appearance in the hepatobiliary phase.
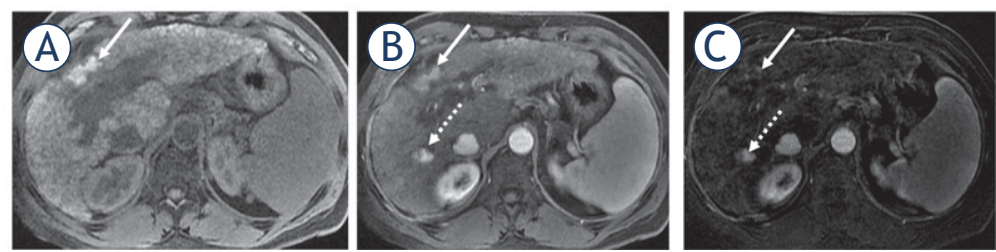

FIGURE 6. 62-year-old man with cirrhosis due to alcohol with a history of percutaneous radiofrequency ablation of a nodule in segment IV. On pre-contrast $\mathrm{Tl}$ weighted image (TIWI) with fat suppression (A) there is high signal intensity within the treated area (arrows) that was maintained in the arterial phase (B); however, on subtraction no enhancement is detected $(C)$, which is compatible with no viable tumor. New HCC appeared during the follow-up in the segment VI (dashed arrow) with a true arterial hyperenhancement.

diagnosis and improving patient outcomes. Most of these cases are challenging and biopsy may be needed for confirmatory diagnosis.

\section{Atypical enhancement patterns}

Hypervascular nodules without washout appearance: Well-differentiated and small HCC lesions may
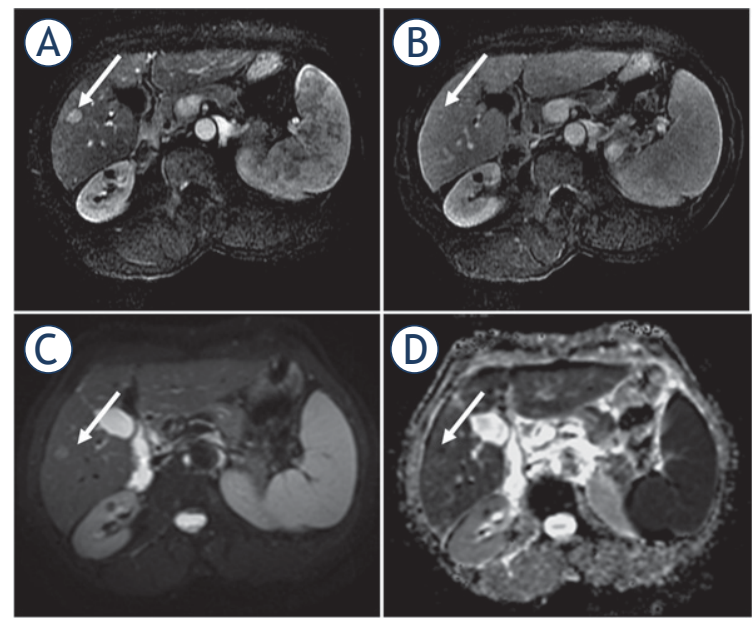

FIGURE 7. 59-year-old woman with cirrhosis due to chronic hepatitis B and small HCC. Small nodule (arrows) in segment $\checkmark$ with arterial phase hyperenhancement (A), without washout appearance (B), and with diffusion restriction (C, D). The patient underwent percutaneous biopsy with the diagnosis of well-differentiated HCC.

show atypical patterns of enhancement with lack of or poor arterial phase enhancement and persistent enhancement in the venous and delayed phases (Figure 7). ${ }^{38,39}$ Differential diagnoses are benign hypervascular lesions (e.g., hemangioma, focal nodular hyperplasia, and adenomas) and hypervascular metastasis.

Hypovascular nodules: Only about $10 \%$ of HCC are hypovascular ${ }^{38}$ and the diagnosis can be challenging. However, in a patient with high risk to develop $\mathrm{HCC}$, hypovascular nodules are suspicious.

\section{Diffuse hepatocellular carcinoma}

Diffuse hepatocellular carcinoma is a rare aggressive form of HCC characterized by poorly defined margins and atypical enhancement patterns (mild heterogeneous enhancement, most commonly hypovascular). Frequently, there is involvement of the portal and hepatic veins with thrombosis (Figure 8)..$^{38}$

\section{Hepatocellular carcinoma in non- cirrhotic liver}

Twenty percent of HCCs may occur in a non-cirrhotic liver. The radiological appearance of such HCCs is larger, well-demarcated, solitary lesions with large areas of necrosis; they are usually diagnosed at a later stage..$^{40}$ 


\section{Differential diagnosis}

Although arterial hyperenhancement is considered the most consistent feature of HCC, it is also present in other non-malignant lesions especially small non-malignant ones, which contributes to the high incidence of false positives.

\section{Vascular disorders}

Transient arterial enhancement due to focal obstruction of a distal parenchymal portal vein or nontumorous arterioportal shunts, for example, is often seen in the cirrhotic liver. Usually, these vascular disorders are peripheral, wedge-shaped lesions, isointense to surrounding parenchyma on pre-contrast images and do not present restricted diffusion or displace internal vasculature. ${ }^{36-38}$

\section{Focal confluent hepatic fibrosis}

Observed in end-stage liver disease, focal confluent hepatic fibrosis can be mass-like and mistaken for HCC once it presents similar low signal intensity relative to the liver on T1WI and hyperintensity on T2WI. However, unlike HCC, it is usually associated with atrophy and capsular retraction of the affected segment, as well as delayed contrast enhancement. ${ }^{36-38}$

\section{Hemangiomas, focal nodular hyperplasia, and hepatic adenomas}

Other benign lesions such as hemangiomas, focal nodular hyperplasia, and hepatic adenomas are rare in the cirrhotic liver, probably because of the process of cirrhosis, and they can be difficult to distinguish from HCC. ${ }^{36-38}$

\section{Intrahepatic cholangiocarcinoma}

Intrahepatic cholangiocarcinoma usually shows rim enhancement with progressive and concentric filling of contrast material in the later phases, which would be an atypical pattern of enhancement for HCC (Figure 9). Other features more commonly associated with intrahepatic cholangiocarcinoma than HCC are intrahepatic biliary duct dilation distal to the tumor and associated capsular retraction. Association with tumoral thrombosis is rare and when narrowing or obstruction of the portal vein occurs, the latter are usually due to external compression. ${ }^{18}$
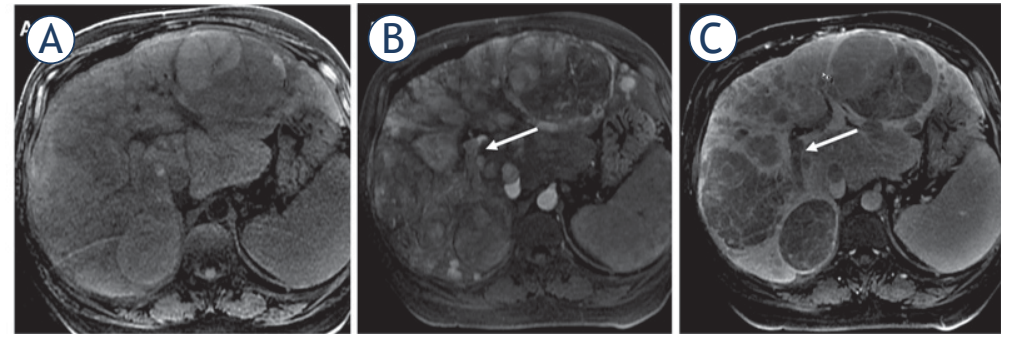

FIGURE 8. 69-year-old man with cirrhosis due to alcohol with diffuse infiltrative HCC (A). Diffuse hepatic mass with areas of hypervascular arterial phase (B) and washout appearance (C), with tumoral thrombus within the right portal vein (arrows).

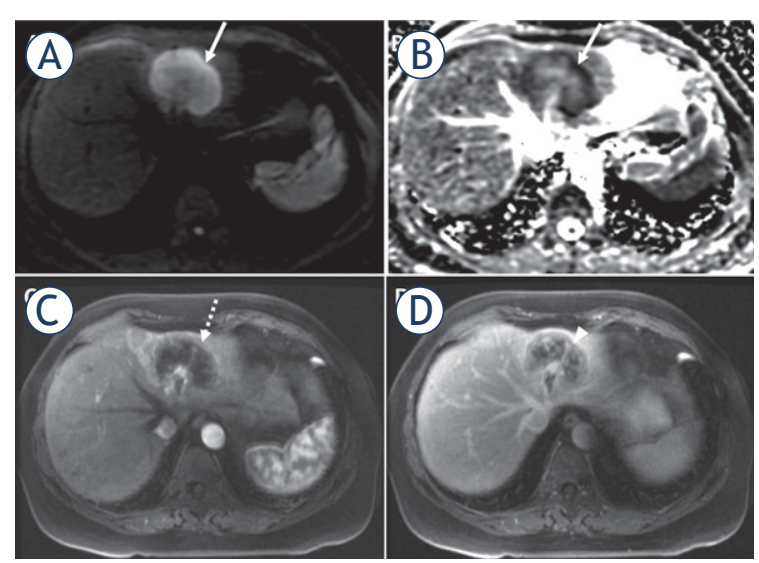

FIGURE 9. 65-year-old woman without liver disease with surgically-proven intrahepatic cholangiocarcinoma confirmed. Nodule in the left hepatic lobe with peripheral restricted diffusion (arrows) (A, B), rim of arterial hyperenhancement (dashed arrow), and peripheral washout appearance (arrowhead).

\section{Hepatocellular-cholangiocarcinoma}

Combined hepatocellular-cholangiocarcinoma (cHCC-CC) is a rare variant of primary hepatic cancer that is clinically and pathologically distinct from pure HCC. Imaging features are variable depending on the predominant histologic component, and although they overlap more frequently with those of cholangiocarcinoma, they can also mimic HCC. ${ }^{18,40,41}$ cHCC-CC may appear hypointense on T1WI and iso to hyperintense on T2WI with or without central hypointense focus, which represents a central cholangiocarcinoma or fibrotic component. ${ }^{19,43}$ On dynamic imaging, early ring enhancement with centripetal progression or heterogeneous early enhancement with partial washout are possible presentations. ${ }^{41}$ On MR imaging with a hepatocellular agent, irregular shape, strong peripheral enhancement, and absence of target sign favor cHCC-CC, particularly the HCC predominant type. ${ }^{42}$ 
TABLE 4. Main classifications used to assess tumor response after locoregional treatment

\begin{tabular}{|c|c|c|c|}
\hline Criteria & System & Response & Definition \\
\hline \multirow[t]{6}{*}{ Size } & WHO & CR & Disappearance of all TL \\
\hline & & PR & $\geq 50 \%$ decrease in $\mathrm{CP}$ of $\mathrm{TL}$ \\
\hline & & SD & $<50 \%$ decrease to $\leq 25 \%$ increase in $\mathrm{CP}$ of $\mathrm{TL}$ \\
\hline & & PD & $>25 \%$ increase from maximum response of TL \\
\hline & & SD & $<30 \%$ decrease to $\leq 20 \%$ increase in $\mathrm{MD}$ of $\mathrm{TL}$ \\
\hline & & PD & $>20 \%$ increase from maximum response of TL \\
\hline \multirow[t]{11}{*}{ Necrosis } & MRECIST & CR & Disappearance of any intratumoral enhancement in all TL \\
\hline & & PR & $\geq 30 \%$ decrease in SMD of enhancing tissue in $\mathrm{TL}$ \\
\hline & and & PR & $\geq 50 \%$ decrease in amount of enhancing tissue in $\mathrm{TL}$ \\
\hline & EASL $_{\text {est }}$ & SD & $<50 \%$ decrease in amount of enhancing tissue in TL \\
\hline & & PD & $>25 \%$ increase in amount of enhancing tissue in TL and/or new enhancement in previously treated lesions \\
\hline & LI-RADS & Nonviable & No suspicious lesion enhancement \\
\hline & & Equivocal & Atypical enhancement not meeting criteria to viable tumor \\
\hline & & Viable & $\begin{array}{l}\text { Nodular, mass-like, or thick irregular tissue in or along the treated lesion with any of the following: arterial phase } \\
\text { hyperenhancement or washout appearance or enhanced similar to pretreatment }\end{array}$ \\
\hline & & & $100 \%$ of tumor necrosis or reduction \\
\hline & RECICL & TE4 & Necrotized area larger than the tumor (enough ablative margin) \\
\hline & & b & Necrotized area similar in size to the tumor (insufficient ablative margin) \\
\hline
\end{tabular}

$\mathrm{CR}=$ complete response, $\mathrm{CP}=$ cross-product, EASL = European Association for the Study of Liver, LI-RADS = Liver Imaging Reporting and Data System, MD = maximum diameter $\mathrm{PD}=$ progressive disease, $\mathrm{PR}=$ partial response, RECIST = Response Evaluation Criteria for Solid Tumors, $\mathrm{mRECIST}=$ modified RECIST, RECICL = Response Evaluation Criteria in Cancer of the Liver, $S D=$ stable disease, $S M D=$ sum of maximum diameters, TE = treatment effect, TL = target lesion(s), WHO = World Health Organization

\section{Hypervascular metastases}

Hypervascular metastases may also be a diagnostic challenge and typically arise from primary neuroendocrine tumors (pancreatic islet cell tumor, carcinoid tumor, and pheochromocytoma), renal cell carcinoma, thyroid carcinoma, choriocarcinoma, and melanoma. ${ }^{43}$ They are generally irregular with indistinct margins and hyperintense on T2WI with a central cystic or necrotic component and with a variable sign on T1WI, depending on the presence of blood, melanin, and other substances that present high signal on this sequence. On dynamic imaging, they show perilesional rim enhancement and irregular washout on delayed images. ${ }^{44}$

\section{MRI after locoregional therapy of HCC}

Surgical resection or transplantation is the standard treatment of HCC. However, most patients are not eligible for resection and the waiting list for liver transplantation is long. Locoregional therapies can be performed as curative treatment, mainly in small HCC, or as a bridge before transplantation. The goal of locoregional therapy is to achieve tumor necrosis. Overall, treated tumors appear with no internal enhancement on postcontrast phases and viable tumors may have areas of arterial phase hyperenhancement with or without washout ap- 
TABLE 5. MRI and CT estimated sensitivity for the detection of hepatocellular carcinoma (HCC)

\begin{tabular}{lccc}
\hline & MRI & CT & CEUS \\
\hline Overall & $82 \%$ & $77 \%$ & $73 \%$ \\
Tumor size $\geq 2 \mathrm{~cm}$ & $96 \%$ & $94 \%$ & $94 \%$ \\
Tumor size $<2 \mathrm{~cm}$ & $66 \%$ & $63 \%$ & $77 \%$ \\
\hline
\end{tabular}

CEUS $=$ contrast-enhanced ultrasound

pearance (Figure 6). ${ }^{44,45}$ Table 4 shows the main systems used to assess tumor response after locoregional treatment.

\section{Diagnostic performance}

The imaging diagnosis of HCC using only the features on dynamic MRI is highly specific. The overall MRI sensitivity for detection of HCC is $81 \%$, against $68 \%$ using CT. ${ }^{46}$ The dynamic contrast enhanced arterial phase is the most sensitive and specific sequence $(>95 \%) .{ }^{47}$

MRI is especially sensitive for the detection of lesions larger than $2 \mathrm{~cm}$. On the other hand, for the detection of small tumors, although MRI still outperforms CT, the sensitivity remains disappointing. ${ }^{48-53}$ This can be explained by the high frequency of atypical enhancement patterns these small lesions present. ${ }^{52}$ Regarding contrast-enhanced ultrasound (CEUS), it is not recommended as a first-line imaging technique, but improvements have been made in the differential diagnosis of cholangiocarcinoma and hepatocellular carcinoma and some studies have shown it to be more specific than CT and MRI for nodules between 10 and 20 mm. ${ }^{12}$ Table 5 summarizes and compares the sensitivity of MRI, CT, and CEUS for the detection of HCC according to tumor size..$^{51}$

As an attempt to improve the performance of MRI among small HCC, the combined use of DWI with conventional dynamic $\mathrm{MRI}^{54}$ as well as the use of contrast agents other than gadolinium-based contrast media have been proposed. ${ }^{55}$ The combination of super-paramagnetic iron oxide particles with gadolinium-based contrast media have been shown to increase the sensitivity for the detection of HCC measuring 1-2 cm to $92 \% .^{56}$

After local therapies, MRI has also shown to be specific but not sensitive for the detection of small foci of recurrent or residual tumor. ${ }^{57,58}$ In this context, DWI has shown promising results for evaluating response to treatment.

\section{Future directions}

\section{Radiomics}

Advances in technology have allowed for quantitative features to be extracted from imaging scans, adding value to clinical decision-making. In oncology, quantitative radiomics features may allow for the assessment of tumor characteristics including cellularity, perfusion, and oxygenation that can help in characterizing tumors characterization, assessing treatment response, and predicting treatment response. Considering that MRI involves different sequences with several physical mechanisms, the use of MRI in radiomics is promising. ${ }^{59}$

\section{Radiogenomics}

Both quantitative and qualitative data extracted from imaging scans can also be correlated with genetic profiles. It has been demonstrated that imaging phenotypes reflect underlying genomics ${ }^{59}$ and can guide the treatment of those patients, which is important in the new era of personalized medicine.

\section{Positron emission tomography (PET)-MRI}

Positron Emission Tomography (PET)-MRI combines high contrast and anatomical resolution from MRI with wide metabolic properties from PET. This technique is promising considering several new radiotracers. PET-MRI could be especially beneficial for evaluating tumor characteristics. ${ }^{60-62}$

While conventional imaging modalities (MRI and CT) are preferable for detecting HCC, PET can offer additional information about functional or metabolic characteristics of the tumor. Several radiotracers have been used to achieve this objective, including 18F-fluorodeoxyglucose (18F-FDG) to estimate glucose consumption and choline labelled with either $11 \mathrm{C}$ (Cho) or 18F (FCho) to reflect cellmembrane metabolism and tumor proliferation. ${ }^{63-65}$

18 F-FDG is the most widely used radiotracer in oncology and has great sensitivity for detecting metastases from most cancers. FDG uptake correlates with the degree of HCC differentiation, with a higher avidity for poorly differentiated HCC. ${ }^{66} \mathrm{On}$ the other hand, choline shows strong avidity for HCC, especially in well and moderately-differentiated tumors. ${ }^{68}$ Some studies showed that a dualtracer PET using FDG and choline has the best performance to detect $\mathrm{HCC}^{66-68}$ as these tracers complement each other in the detection of HCC based on its histological differentiation. This combination 
may also be a prognostic indicator, with worst outcomes associated with FDG-PET captation. ${ }^{68}$

\section{Perfusion MRI}

Perfusion MRI is a function imaging technique that can provide quantitative data regarding tumor microvasculature. Several studies demonstrated that perfusion MRI can assess tumor response after locoregional therapies, such as transcatheter arterial chemoembolization and radiofrequency ablation. ${ }^{69,70}$ Perfusion MRI can detect vasculature changes of HCC before and after therapy. It is a promising tool in the diagnosis of HCC, as it can be used to target lesions for therapy, to evaluate the efficacy of the treatments and to evaluate recurrence. ${ }^{69-71}$

\section{Conclusions}

In summary, MRI is an essential imaging modality in the diagnostic arsenal of HCC and is especially indicated for the evaluation of small lesions, unclear lesions on $\mathrm{CT}$, and lesions after locoregional therapies. Considering the multiple sequences included on MRI, there is a huge potential to extract several imaging biomarkers that reflect the tumor microenvironment and which, in the future, may add decision-making value in the management of patients with HCC.

\section{Acknowledgments}

The authors would like to thank Joanne Chin for her editorial support on this manuscript.

This research was funded in part through the NIH/NCI Cancer Center Support Grant P30 CA008748. The work by Romina Grazia Giancipoli was partially supported by a scholarship awarded by ISSNAF Imaging Science Chapter.

\section{References}

1. Ferenci $P$, Fried $M$, Labrecque $D$, Bruix J, Sherman $M$, Omata $M$, et al. Hepatocellular carcinoma (HCC): a global perspective. J Clin Gastroenterol 2010; 44: 239-45. doi: 10.1097/MCG.0b013e3181d46ef2

2. Torre LA, Bray F, Siegel RL, Ferlay J, Lortet-Tieulent J, Jemal A. Global cancer statistics 2012. CA Cancer J Clin 2015; 65: 87-108. doi: 10.3322/caac.21262

3. Siegel RL, Miller KD, Jemal A. Cancer statistics, 2018. CA Cancer J Clin 2018; 68: 7-30. doi: 10.3322/caac. 21442

4. Tang A, Hallouch O, Chernyak V, Kamaya A, Sirlin CB. Epidemiology of hepatocellular carcinoma: target population for surveillance and diagnosis. Abdom Radiol (NY) 2018; 43: 13-25. doi: 10.1007/s00261-017-1209-1
5. Krinsky GA, Lee VS. MR imaging of cirrhotic nodules. Abdom Imaging 2000; 25: 471-82.

6. Nishida N, Goel A. Genetic and epigenetic signatures in human hepatocellular carcinoma: a systematic review. Curr Genomics 2011; 12: 130-7. doi: $10.2174 / 138920211795564359$

7. Choi JY, Lee JM, Sirlin CB. CT and MR imaging diagnosis and staging of hepatocellular carcinoma: part I. Development, growth, and spread: key pathologic and imaging aspects. Radiology 2014; 272: 635-54. doi: 10.1148/ radiol.14132361.

8. Crissien AM, Frenette C. Current management of hepatocellular carcinoma. Gastroenterol Hepatol (NY) 2014; 10: 153-61. PMCID: PMC4014047

9. European Association For The Study Of The Liver, European Organisation For Research And Treatment Of Cancer. EASL-EORTC clinical practice guidelines: management of hepatocellular carcinoma. J Hepatol 2012; 56: 908-43. doi: 10.1016/j.jhep.2011.12.001

10. Elsayes KM, Hooker JC, Agrons MM, Kielar AZ, Tang A, Fowler KJ, et al. 2017 Version of LI-RADS for CT and MR imaging: an update. Radiographics 2017 37: 1994-2017. doi: 10.1148/rg.2017170098.

11. Arif-Tiwari H, Kalb B, Chundru S, Sharma P, Costello J, Guessner RW, et al. MRI of hepatocellular carcinoma: an update of current practices. Diagn Interv Radiol 2014; 20: 209-21. doi: 10.5152/dir.2014.13370.

12. Heimbach JK, Kulik LM, Finn RS, Sirlin CB, Abecassis MM, Roberts LR, et al. AASLD guidelines for the treatment of hepatocellular carcinoma. Hepatology 2018; 67: 358-80. doi: 10.1002/hep.29086.

13. Wald C, Russo MW, Heimbach JK, Hussain HK, Pomfret EA, Bruix J. New OPTN/UNOS policy for liver transplant allocation: standardization of liver imaging, diagnosis, classification, and reporting of hepatocellular carcinoma. Radiology 2013; 266: 376-82. doi: 10.1148/radiol.12121698

14. Kambadakone AR, Fung A, Gupta RT, et al. LI-RADS technical requirements for CT, MRI, and contrast-enhanced ultrasound. Abdom Radiol (NY) 2018; 43: 56-74. doi: 10.1007/s00261-017-1345-7.

15. Le Moigne F, Durieux M, Bancel B, Boublay N, Boussel L, Ducerf C, et al. Impact of diffusion-weighted MR imaging on the characterization of small hepatocellular carcinoma in the cirrhotic liver. Magn Reson Imaging 2012; 30: 656-65. doi: 10.1016/j.mri.2012.01.002

16. Mannelli L, Nougaret S, Vargas HA, Do RK. Advances in diffusion-weighted imaging. Radiol Clin North Am 2015; 53: 569-81. doi: 10.1016/j. rcl.2015.01.002

17. Mannelli L, Bhargava P, Osman SF, Raz E, Moshiri M, Laffi G, et al. Diffusionweighted imaging of the liver: a comprehensive review. Curr Probl Diagn Radiol 2013; 42: 77-83. doi: 10.1067/j.cpradiol.2012.07.001

18. Horvat N, Nikolovski I, Long N, Gerst S, Zheng J, Pak LM, et al. Imaging features of hepatocellular carcinoma compared to intrahepatic cholangiocarcinoma and combined tumor on MRI using liver imaging and data system (LI-RADS) version 2014. Abdom Radiol (NY) 2018; 43: 169-78. doi: 10.1007/ s00261-017-1261-x

19. Mannelli L, Kim S, Hajdu CH, Babb JS, Taouli B. Serial diffusion-weighted MR in patients with hepatocellular carcinoma: prediction and assessment of response to transarterial chemoembolization. Preliminary experience. Eur $J$ Radiol 2013; 82: 577-82. doi: 10.1016/j.ejrad.2012.11.026

20. Chegai F, Merolla S, Greco L, Nezzo M, Mannelli L, Orlacchio A. Re: Baseline and early MR apparent diffusion coefficient quantification as a predictor of response of unresectable hepatocellular carcinoma to doxorubicin drugeluting bead chemoembolization. J Vasc Interv Radiol 2016; 27: 1456-8. doi: 10.1016/j.jvir.2016.05.007

21. Gluskin JS, Chegai F, Monti S, Squillaci E, Mannelli L. Hepatocellular carcinoma and diffusion-weighted MRI: detection and evaluation of treatment response. J Cancer 2016; 7: 1565-70. doi: 10.7150/jca.14582

22. Park MS, Kim S, Patel J, Hajdu CH, Do RK, Mannelli L, et al. Hepatocellula carcinoma: detection with diffusion-weighted versus contrast-enhanced magnetic resonance imaging in pretransplant patients. Hepatology 2012; 56: 140-8. doi: 10.1002/hep.25681

23. Agostini A, Kircher MF, Do R, Borgheresi A, Monti S, Giovagnoni A, et al. Magnetic resonance imaging of the liver (including biliary contrast agents) part 1: technical considerations and contrast materials. Semin Roentgenol 2016; 51: 308-16. doi: 10.1053/j.ro.2016.05.015

24. Ramalho J, Ramalho M. Gadolinium deposition and chronic toxicity. Magn Reson Imaging Clin N Am 2017; 25: 765-78. doi: 10.1016/j.mric.2017.06.007 
25. Hernando D, Levin YS, Sirlin CB, Reeder SB. Quantification of liver iron with MRI: state of the art and remaining challenges. J Magn Reson Imaging 2014; 40: 1003-21. doi: 10.1002/jmri.24584

26. Agostini A, Kircher MF, Do RK, Borgheresi A, Monti S, Giovagnoni A. Magnetic resonanance imaging of the liver (including biliary contrast agents) part 2: protocols for liver magnetic resonanance imaging and characterization of common focal liver lesions. Semin Roentgenol 2016; 51 : 317-33. doi: 10.1053/j.ro.2016.05.016

27. Liu D, Song B, Huang ZX, Wu B, Tang HH. [The iron content of hepatocellular carcinoma associated nodules: study of histopathology and MR imaging]. [Chinese]. Sichuan Da Xue Xue Bao Yi Xue Ban 2016; 47: 376-81. PMID: 27468484

28. Siripongsakun S, Lee JK, Raman SS, Tong MJ, Sayre J, Lu DS. MRI detection of intratumoral fat in hepatocellular carcinoma: potential biomarker for a more favorable prognosis. AJR Am J Roentgenol 2012; 199: 1018-25. doi: 10.2214/AJR.12.8632

29. Singh S, Venkatesh SK, Loomba R, Wang Z, Sirlin C, Chen J, et al. Magnetic resonance elastography for staging liver fibrosis in non-alcoholic fatty liver disease: a diagnostic accuracy systematic review and individual participant data pooled analysis. Eur Radiol 2016; 26: 1431-40. doi: 10.1007/s00330015-3949-z

30. Thompson SM, Wang J, Chandan VS, Glaser KJ, Roberts LR, Ehman RL, et al. MR elastography of hepatocellular carcinoma: correlation of tumor stiffness with histopathology features-preliminary findings. Magn Reson Imaging 2017; 37: 41-5. doi: 10.1016/j.mri.2016.11.005

31. Gordic S, Ayache JB, Kennedy P, Besa C, Wagner M, Bane O, et al. Value of tumor stiffness measured with MR elastography for assessment of response of hepatocellular carcinoma to locoregional therapy. Abdom Radiol (NY) 2017; 42: 1685-94. doi: 10.1007/s00261-017-1066-y

32. Hanna RF, Aguirre DA, Kased N, Emery SC, Peterson MR, Sirlin CB. Cirrhosisassociated hepatocellular nodules: correlation of histopathologic and MR imaging features. Radiographics 2008; 28: 747-69. doi: 10.1148/ rg.283055108

33. Efremidis SC, Hytiroglou P. The multistep process of hepatocarcinogenesis in cirrhosis with imaging correlation. Eur Radiol 2002; 12: 753-64. doi: $10.1007 / \mathrm{s} 00330-001-1142-z$

34. Efremidis SC, Hytiroglou P, Matsui O. Enhancement patterns and signalintensity characteristics of small hepatocellular carcinoma in cirrhosis: pathologic basis and diagnostic challenges. Eur Radiol 2007; 17: 2969-82. doi: 10.1007/s00330-007-0705-z

35. Jeong YY, Yim NY, Kang HK. Hepatocellular carcinoma in the cirrhotic liver with helical CT and MRI: imaging spectrum and pitfalls of cirrhosisrelated nodules. AJR Am J Roentgenol 2005; 185: 1024-32. doi: 10.2214/ AJR.04.1096

36. Sadek AG, Mitchell DG, Siegelman ES, Outwater EK, Matteucci T, Hann HW. Early hepatocellular carcinoma that develops within macroregenerative nodules: growth rate depicted at serial MR imaging. Radiology 1995; 195753-6. doi: 10.1148/radiology.195.3.7754006

37. Willatt JM, Hussain HK, Adusumilli S, Marrero JA. MR Imaging of hepatocellular carcinoma in the cirrhotic liver: challenges and controversies. Radiology 2008; 247: 311-30. doi: 10.1148/radiol.2472061331

38. Roumanis PS, Bhargava P, Kimia Aubin G, Choi Jl, Demirjian AN, Thayer DA, et al. Atypical magnetic resonance imaging findings in hepatocellular carcinoma. Curr Probl Diagn Radiol 2015; 44: 237-45. doi: 10.1067/j.cpradiol.2014.03.002

39. Mannelli L, Hoang MV, Sabath AP, Linnau KF. An unusual oral mass. Gastroenterology 2012; 142: e14-5. doi.org/10.1053/j.gastro.2011.08.047

40. Trevisani F, Frigerio M, Santi V, Grignaschi A, Bernardi M. Hepatocellular carcinoma in non-cirrhotic liver: a reappraisal. Dig Liver Dis 2010; 42: 341-7. doi: 10.1016/j.dld.2009.09.002

41. Potretzke TA, Tan BR, Doyle MB, Brunt EM, Heiken JP, Fowler KJ. Imaging features of biphenotypic primary liver carcinoma (hepatocholangiocarcinoma) and the potential to mimic hepatocellular carcinoma: LI-RADS analysis of CT and MRI features in 61 cases. AJR Am J Roentgenol 2016; 207: 25-31. doi: 10.2214/AJR.15.14997

42. Maximin S, Ganeshan DM, Shanbhogue AK, Dighe MK, Yeh MM, Kolokythas $\mathrm{O}$, et al. Current update on combined hepatocellular-cholangiocarcinoma. Eur J Radiol Open 2014; 1: 40-8. doi: 10.1016/j.ejro.2014.07.001
43. Mannelli L, Monti S, Grieco V, Matesan M. Hepatic lesions in a cirrhotic liver: primary or metastases? J Nucl Med Technol 2017; 45: 50-2. doi: 10.2967/ jnmt.116.183228.

44. Khosa F, Khan AN, Eisenberg RL. Hypervascular liver lesions on MRI. AJR Am J Roentgenol 2011; 197: W204-20. doi: 10.2214/AJR.10.5382

45. Ayuso C, Rimola J, Garcia-Criado A. Imaging of HCC. Abdom Imaging 2012 37: 215-30. doi: 10.1007/s00261-011-9794-x

46. Mannelli L, Kim S, Hajdu CH, Babb JS, Clark TW, Taouli B. Assessment of tumor necrosis of hepatocellular carcinoma after chemoembolization: diffusion-weighted and contrast-enhanced MRI with histopathologic correlation of the explanted liver. AJR Am J Roentgenol 2009; 193: 1044-52. doi: 10.2214/AJR.08.1461

47. Colli A, Fraquelli M, Casazza G, Massironi S, Colucci A, Conte D, et al. Accuracy of ultrasonography, spiral CT, magnetic resonance, and alpha-fetoprotein in diagnosing hepatocellular carcinoma: a systematic review. Am J Gastroenterol 2006; 101: 513-23. doi: 10.1111/j.1572-0241.2006.00467.x

48. Becker-Weidman DJ, Kalb B, Sharma P, Kitajima HD, Lurie CR, Chen Z, et al. Hepatocellular carcinoma lesion characterization: single-institution clinical performance review of multiphase gadolinium-enhanced MR imaging-comparison to prior same-center results after MR systems improvements. Radiology 2011; 261: 824-33. doi: 10.1148/radiol.11110157

49. Krinsky GA, Lee VS, Theise ND, Weinreb JC, Morgan GR, Diflo T, et al. Transplantation for hepatocellular carcinoma and cirrhosis: sensitivity of magnetic resonance imaging. Liver Transp/ 2002; 8: 1156-64. doi: 10.1053/ jlts.2002.35670

50. Burrel M, Llovet JM, Ayuso C, Iglesias C, Sala M, Miquel R, et al. MRI angiography is superior to helical CT for detection of HCC prior to liver transplantation: an explant correlation. Hepatology 2003; 38: 1034-42. doi: 10.1053/ jhep.2003.50409

51. Lee YJ, Lee JM, Lee JS, Lee HY, Park BH, Kim YH, et al. Hepatocellular carcinoma: diagnostic performance of multidetector CT and MR imaging-a systematic review and meta-analysis. Radiology 2015; 275: 97-109. doi: 10.1148/radiol.14140690

52. Forner A, Vilana R, Ayuso C, Bianchi L, Solé M, Ayuso JR, et al. Diagnosis of hepatic nodules $20 \mathrm{~mm}$ or smaller in cirrhosis: Prospective validation of the noninvasive diagnostic criteria for hepatocellular carcinoma. Hepatology 2008; 47: 97-104. doi: 10.1002/hep.21966

53. Chou R, Cuevas C, Fu R, Devine B, Wasson N, Ginsburg A, et al. Imaging techniques for the diagnosis of hepatocellular carcinoma: a systematic review and meta-analysis. Ann Intern Med 2015; 162: 697-711. doi: 10.7326/ M14-2509

54. Xu PJ, Yan FH, Wang JH, Shan Y, Ji Y, Chen CZ. Contribution of diffusionweighted magnetic resonance imaging in the characterization of hepatocellular carcinomas and dysplastic nodules in cirrhotic liver. J Comput Assist Tomogr 2010; 34: 506-12. doi: 10.1097/RCT.0b013e3181da3671

55. Kierans AS, Kang SK, Rosenkrantz AB. The diagnostic performance of dynamic contrast-enhanced MR imaging for detection of small hepatocellular carcinoma measuring up to $2 \mathrm{~cm}$ : a meta-analysis. Radiology 2016; 278: 82-94. doi: 10.1148/radiol.2015150177

56. Ward J, Guthrie JA, Scott DJ, Atchley J, Wilson D, Davies MH, et al. Hepatocellular carcinoma in the cirrhotic liver: double-contrast MR imaging for diagnosis. Radiology 2000; 216: 154-62. doi: 10.1148/ radiology.216.1.ro0j|24154

57. Lu DS, Yu NC, Raman SS, Limanond P, Lassman C, Murray K, et al. Radiofrequency ablation of hepatocellular carcinoma: treatment success as defined by histologic examination of the explanted liver. Radiology 2005; 234: 954-60. doi: 10.1148/radiol.2343040153

58. Buscarini L, Buscarini E, DiStasi M, Vallisa D, Quaretti P, Rocca A. Percutaneous radiofrequency ablation of small hepatocellular carcinoma: long-term results. Eur Radiol 2001; 11: 914-21. doi: 10.1007/s003300000659

59. Kuo MD, Gollub J, Sirlin CB, Ooi C, Chen X. Radiogenomic analysis to identify imaging phenotypes associated with drug response gene expression programs in hepatocellular carcinoma. J Vasc Interv Radiol 2007; 18: 821-31. doi: 10.1016/j.jvir.2007.04.031

60. Jadvar H, Colletti PM. Competitive advantage of PET/MRI. Eur J Radiol 2014; 83: 84-94. doi: 10.1016/j.ejrad.2013.05.028 
61. Kong $\mathrm{E}$, Chun KA, Cho IH. Quantitative assessment of simultaneous F-18 FDG PET/MRI in patients with various types of hepatic tumors: correlation between glucose metabolism and apparent diffusion coefficient. PLoS One 2017; 12: e0180184. doi: 10.1371/journal.pone.0180184

62. Sun L, Wu H, Guan YS. Positron emission tomography/computer tomography: challenge to conventional imaging modalities in evaluating primary and metastatic liver malignancies. World J Gastroenterol 2007; 13: 2775-83.

63. Talbot JN, Gutman F, Fartoux L, Grange JD, Ganne N, Kerrou K, et al. PET/CT in patients with hepatocellular carcinoma using [(18)F]fluorocholine: preliminary comparison with [(18)F]FDG PET/CT. Eur J Nucl Med Mol Imaging 2006; 33: 1285-9. doi: 10.1007/s00259-006-0164-9

64. Mertens K, Slaets D, Lambert B, Acou M, De Vos F, Goethals I. PET with (18)F-labelled choline-based tracers for tumour imaging: a review of the literature. Eur J Nucl Med Mol Imaging 2010; 37: 2188-93. doi: 10.1007/ s00259-010-1496-z

65. Yang SH, Suh KS, Lee HW, et al. The role of (18)F-FDG-PET imaging for the selection of liver transplantation candidates among hepatocellular carcinoma patients. Liver Transp/ 2006; 12: 1655-60. doi: 10.1002/It.20861

66. Bertagna F, Bertoli M, Bosio G, Biasiotto G, Sadeghi R, Giubbini R, et al. Diagnostic role of radiolabelled choline PET or PET/CT in hepatocellular carcinoma: a systematic review and meta-analysis. Hepatol Int 2014; 8 493-500. doi: 10.1007/s12072-014-9566-0

67. Ho CL, Chen S, Yeung DW, Cheng TK. Dual-tracer PET/CT imaging in evaluation of metastatic hepatocellular carcinoma. J Nucl Med 2007; 48: 902-9. doi: $10.2967 /$ jnumed.106.036673

68. Castilla-Lièvre MA, Franco D, Gervais $P$, Kuhnast $B$, Agostini $H$, Marthey $\mathrm{L}$, et al. Diagnostic value of combining ${ }^{11} \mathrm{C}$-choline and ${ }^{18} \mathrm{~F}$-FDG PET/CT in hepatocellular carcinoma. Eur J Nucl Med Mol Imaging 2016; 43: 852-9. doi: $10.1007 / \mathrm{s} 00259-015-3241-0$

69. Shao GL, Zheng JP, Guo LW, Chen YT, Zeng H, Yao Z. Evaluation of efficacy of transcatheter arterial chemoembolization combined with computed tomography-guided radiofrequency ablation for hepatocellular carcinoma using magnetic resonance diffusion weighted imaging and computed tomography perfusion imaging: A prospective study. Medicine (Baltimore) 2017; 96: e5518. doi: 10.1097/MD.0000000000005518

70. Chen X, Xiao E, Shu D, Yang C, Liang B, He Z, et al. Evaluating the therapeutic effect of hepatocellular carcinoma treated with transcatheter arterial chemoembolization by magnetic resonance perfusion imaging. Eur J Gastroenterol Hepatol 2014; 26: 109-13. doi: 10.1097/MEG.0b013e328363716e

71. Bayle $\mathrm{M}$, Clerc-Urmès I, Ayav A, Bronowicki JP, Petit I, Orry $\mathrm{X}$, et al Computed tomographic perfusion with $160-\mathrm{mm}$ coverage: comparative analysis of hepatocellular carcinoma treated by two transarterial chemoembolization courses relative to magnetic resonance imaging findings. Abdom Radiol (NY) 2018. doi: 10.1007/s00261-018-1714-x 